\title{
Implicancias del uso de nonoxinol en el Uruguay
}

\author{
Míguez, D. ${ }^{(1)}$ \\ Contacto: dmiguez@latu.org.uy \\ (1) Departamento de Aguas y Productos Químicos - Laboratorio Tecnológico del Uruguay (LATU)
}

\begin{abstract}
Resumen
Los disruptores endócrinos interfieren con la actividad de las hormonas en el organismo, imitando o bloqueando a las naturales. Son ubicuas y bioacumulables. Los que interfieren con estrógenos pueden provocar cáncer o una merma de la capacidad reproductora, además de cambios en el sexo de los peces y una afectación de la biodiversidad. En nuestro país aun se están consumiendo sustancias han sido prohibidas a nivel mundial por causar estos efectos. Así por ejemplo, desde enero de 2005 en Europa se ha prohibido productos con más de $0,1 \%$ de nonilfenol polietoxilado. En Uruguay se usa cada vez más, como agente de limpieza industrial (lavaderos de lana, curtiembres), institutional y doméstica (polvos de baja espuma, limpiadores). Es poco biodegradable y llega a los cursos de agua, sus sedimentos y a los seres vivos. Se debería tomar conciencia de la gravedad de este asunto y utilizar los sucedáneos para prevenir estas consecuencias.
\end{abstract}

\begin{abstract}
Endocrine disruptors interfere with the hormonal activity in the organism, mimicking or blocking natural hormones. They are ubiquitous and bioaccumulative. These substances interfere with estrogens and may cause cancer or a decrease in reproductive capacity in addition to changes in the sex of fish and an affectation of biodiversity. In our country we are still consuming substances that have been prohibited globally for causing these effects. For example, since January 2005 in Europe has banned products with more than $0.1 \%$ of nonylphenol polyethoxylated. In Uruguay, it is increasingly used as an industrial cleaning agent (wool washing, tanneries), institutional and domestic cleaning (low foam powdered detergents, cleaners). It has low biodegradability and reaches the waterways, sediments and living beings. We should grasp the seriousness of this matter and use substitutes to prevent these consequences.
\end{abstract}

\section{La Directiva Europea}

$\mathrm{E}_{\mathrm{a}}^{\mathrm{n}}$ enero de 2005 entró a regir en Europa la prohibición para a venta y uso de productos con más de $0,1 \%$ de nonilfenol polietoxilado y de nonilfenol (Directiva 2003/53/EC), excepto en el caso de sistemas de aplicación cerrados. Esto se decidió luego que la evaluación de riesgos ambientales mostraron su alta toxicidad acuática y su incompleta biodegradabilidad. Se espera que en el futuro haya una disminución en las emisiones hacia el medio acuático cercana a $80 \%$.

\section{Destino, persistencia y concentraciones en los compartimientos ambientales}

Existen estudios realizados en los países escandinavos que determinan la concentración en lodos de tratamiento $(26-1100 \mathrm{mg} / \mathrm{kg})$ y en moluscos $(0,20-0,40 \mu \mathrm{g} / \mathrm{g})$. Las investigaciones han demostrado que los alquilfenoles mono o di-etoxilados y los alquilfenoles son estrogénicos para los peces, los pájaros y los mamíferos y actúan en concentraciones extremadamente bajas. Durante los procedimientos convencionales de tratamiento de aguas residuales, los polietoxilatos se degradan aeróbicamente a alquilfenol que es más tóxico que el compuesto original. Después de transcurridas 135 horas hay solamente un $45 \%$ de degradación de los productos químicos.

Nota: Este estudio fue hecho también en el LATU por respirometría por la autora en base a la norma ISO 9408, con resultados semejantes. En otro estudio hecho en nuestro laboratorio, se observó que los alcoholes grasos etoxilados, que no son tóxicos y pueden usarse como sucedáneos, pero que son algo más caros, se biodegradan en un porcentaje mayor a $90 \%$.

Las diferencias en la eficiencia de tratamiento de cada compuesto y los productos de degradación entre las plantas pueden ser atribuidas a las cargas iniciales, los diseños y las condiciones operativas, así como a la temperatura de los tratamientos. Los compartimientos ambientales hacia los cuales se liberan estos compuestos son las aguas superficiales (ríos, lagos, mares y sus sedimentos) a través de las plantas de tratamiento, a los suelos a través de los lodos cuando se los dispersa sobre la tierra y también en el aire.

\section{Bioacumulación y actividad disruptora hormonal en el ambiente}

A causa de su naturaleza lipofílica, se acumulan en los lodos de tratamiento y en los sedimentos y se bioacumulan en las especies acuáticas, tanto de agua dulce como especialmente en los peces de agua salada. El nonilfenol está considerado como una sustancia química disruptora endócrina que induce la producción de vitelogenina en las truchas macho, lo cual es un proceso que ocurre normalmente en peces hembra como respuesta a los estrógenos en el ciclo reproductivo. Induce el desarrollo precoz de los ovarios y cambios de sexo en algunas especies de peces. Suele aparecer un aparato ovipositor (para puesta de huevos) en el macho. Es también estrogénico para las ratas y simula los efectos del estradiol y tiene actividad uterotrófica mediada por el receptor estrogénico. A causa de su presencia en el ambiente y su actividad estrogénica puede estar involucrado en los cambios en la reproducción observados en la naturaleza, así como la pérdida de la biodiversidad y la disminución de la pesca, ya que causa disfunciones en la espermatogénesis en los peces.

\section{Toxicidad y acciones sobre la salud humana}

Si bien su toxicidad aguda es moderada (DL50 580 a $2462 \mathrm{mg} / \mathrm{kg}$ en ratas) para la exposición oral, se lo clasifica como corrosivo y peligroso. En los pasados 30-50 años el número de espermatozoi- 
des promedio en poblaciones humanas ha disminuido a la mitad. El aumento de disfunciones reproductivas observadas en los hombres se puede vincular a la exposición durante su vida fetal.

\section{Producción y usos}

\section{Import of NPE trend in Uruguay}

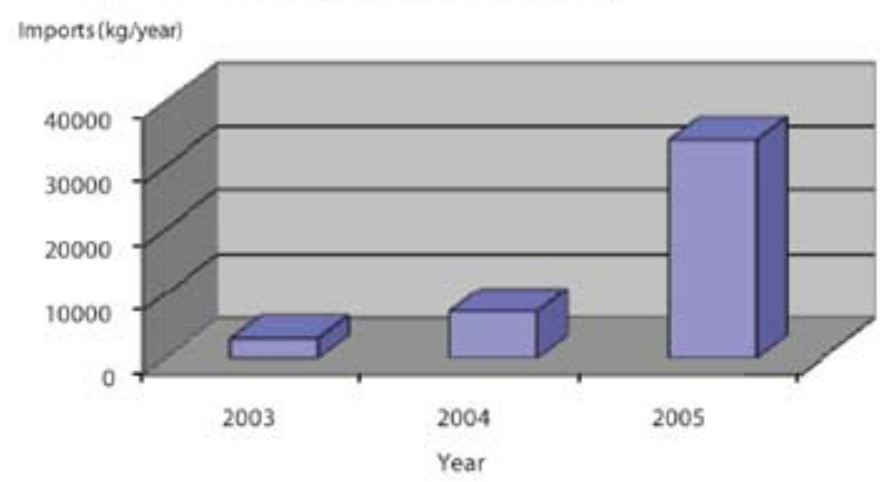

Figura 1. Tendencia de importaciones de estos productos en Uruguay

Antes de las restricciones, el nonilfenol era producido en toneladas y su demanda se incrementaba cerca de un 2 por ciento anual. Se usa como emulsificantes, agentes dispersores, tensioactivos o agentes de mojado. Su uso principal es para limpieza industrial, institutional y doméstica. Otros sectores significativos es para la polimerización en emulsión (12\%), los textiles (10\%), la síntesis orgánica $(9 \%)$ y el cuero (8\%). El uso estimado en Europa Occidental en 1997 fue de 76600 toneladas.

En Uruguay, el uso de nonoxinol se está extendiendo y aumentando en forma drástica. La gráfica de más abajo muestra la tendencia de las importaciones de la base para detergentes de nonilfenol polietoxilado. Se usa para la extracción de grasas, lavado de lana, en la industria textil y la del cuero, pero también forman parte de variados limpiadores domésticos y polvos de lavado de baja espuma, que finalizan en las aguas de los estuarios, ríos y arroyos. En las licitaciones de productos se ve frecuentemente en las especificaciones de compra que se solicita este compuesto.

\section{Posibles riesgos para la salud ambiental en Uruguay}

Uruguay tiene la más alta tasa de cáncer en América Latina. La tendencia en mortalidad por cáncer en el Uruguay en el período entre 1953 y 1997 fue evaluada por Barrios et al (2002). El cáncer prostático se incrementó en todo el período. La tasa de cáncer mamario aumentó significativamente y los de ovario han comenzado a mostrar también más casos.

Los hábitos alimentarios y el de fumar también pueden ser factores causales, pero, de todos modos, deberían hacerse todos los esfuerzos como para disminuir los riesgos asociados a las sustancias sospechosas de causar esas enfermedades. La legislación nacional debería actualizarse para incluir estas sustancias. Las plantas de tratamiento, cuando existen, muchas veces operan en forma ineficiente y por lo tanto los contaminantes terminan en los cursos de agua, llegan a los sedimentos y permanecen como nonilfenol en la fracción no polar o pueden bioacumularse en el tejido lipídico de la biota y magnificarse al ascender en la cadena alimentaria acuática. La posibilidad de encontrar este compuesto en el agua potable no ha sido investigada en nuestro país. Además de su uso en preparaciones de limpieza, el contacto dérmico ocurre en forma directa cuando se lo usa como espermaticida.

\section{Conclusiones}

$\mathrm{E}^{\mathrm{s}}$ un hecho muy notorio que en lugar de reducirse la demanda y el uso del nonilfenol y sus derivados etoxilados, en nuestro país ha habido un enorme incremento en los últimos años, mientras concomitantemente a la prohibición realizada por otros países. Algunos de los importadores son de países limítrofes, pero la mayor cantidad entra al país desde China, Europa y los Estados Unidos. Quisiera hacer un llamado a trabajar para tener una política globalizada en la producción y exportaciones de productos peligrosos, ya que el mundo es uno solo y el medio ambiente no conoce fronteras. Los peces que capturamos y comemos en América del Sur pueden eventualmente estar alimentando a las aves migratorias que llegarán al Hemisferio Norte. Las compañías no deberían producir esta sustancia y debería existir una armonización de las legislaciones ambientales internacionales en el futuro. Debería organizarse una campaña de monitoreo de estas sustancias y otras que ejercen actividad estrogénica.

El sector industrial todavía no ha tomado conciencia de la gravedad de este asunto. Hay sucedáneos que se pueden usar, tales como los alcoholes grasos polietoxilados y otros tensioactivos no nocivos, que tienen similares propiedades funcionales. Desafortunadamente, el bajo precio del producto parece ser un factor más convincente que los riesgos. Desde el punto de vista ético, el rédito económico no debería estar en la misma escala de valores éticos donde se ubican la salud humana y ambiental.

\section{Referencias}

Barrios E. /et al/. 2002. Tendencias de la mortalidad por cáncer en Uruguay 1953-1997. Rev. Méd. Urug. 18(2): 167-174

Christiansen T. ; Korsgaard B.; Jespersen A. 1998. Effects of nonylphenol and 17beta-oestradiol on vitellogenin synthesis, testicular structure and cytology in male eelpout Zoarces viviparus. En: Journal of Experimental Biology. 201(2): 179-192.

European Union. Directive 2003/53/EC of the European Parliament and of the Council of 18 June 2003 amending for the 26th time Council Directive 76/769/EEC relating to restrictions on the marketing and use of certain dangerous substances and preparations (nonylphenol, nonylphenol ethoxylate and cement). Disponible en: http://eur-lex.europa.eu/pri/en/oj/ dat/2003/1_178/1_17820030717en00240027.pdf

Lee P-C. ; Lee W. 1996. In vivo estrogenic action of nonylphenol in immature female. En: Rats Bulletin of Environmental Contamination and Toxicology. 57(3): 341-348

Maguire R. J. 1999. Review of the persistence of nonylphenol and nonylphenol ethoxylates in aquatic environments. Water Quality Research Journal of Canada. 34(1): 37-78.

Nordic steering group for assessment of health effects of chemicals. 1999. Health effects of selected chemicals 4-5. Nord (15): 303-16

OSPAR Commision. Nonylphenol/Nonylphenolethoxylates. London OSPAR, 2001. (Hazardous Substances Series)

Sharpe R. M.; Skakkebæk N.E. 1993. Are oestrogens involved in falling sperm counts and disorders of the male reproductive tract? En: Lancet. (341): 1392-1395.

Silicon Valley Toxics Coalition. Disponible en: http://www.svtc.org

United States Environmental Protection Agency. Ambient aquatic life water quality criteria for nonylphenol - Draft. EPA 822-R-03-029. Disponible en: http://www.epa.gov/waterscience/criteria/nonylphenol/draft-doc.pdf

Wahlberg C.; Renberg L. 1990. Wideqvist U determination of NP and NP ethoxylates as their pentafluorobenzoates in water, sewage sludge and biota. En: Chemosphere 20(1-2): 179-196. 